\title{
Spatiotemporal variability of soil nutrients and the responses of growth during growth stages of winter wheat in northern China
}

\author{
Baowei Su${ }^{1}$, Gengxing Zhao ${ }^{1}$, Chao Dong ${ }^{2}$ \\ 1 College of Resources and Environment, Shandong Agricultural University, Tai'an, China, 2 College of \\ Information Science and Engineering, Shandong Agricultural University, Tai'an, China \\ *zhaogx@sdau.edu.cn
}

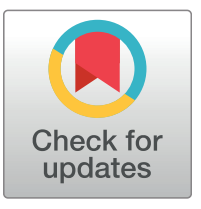

\section{G OPEN ACCESS}

Citation: Su B, Zhao G, Dong C (2018) Spatiotemporal variability of soil nutrients and the responses of growth during growth stages of winter wheat in northern China. PLoS ONE 13(12): e0203509. https://doi.org/10.1371/journal. pone.0203509

Editor: Yuan Huang, Huazhong Agriculture University, CHINA

Received: August 12, 2018

Accepted: November 13, 2018

Published: December 4, 2018

Copyright: @ 2018 Su et al. This is an open access article distributed under the terms of the Creative Commons Attribution License, which permits unrestricted use, distribution, and reproduction in any medium, provided the original author and source are credited.

Data Availability Statement: All relevant data are within the manuscript and its Supporting Information files.

Funding: This study was supported jointly by the twelfth Five-Year National Science and Technology Support Plan (No.2015BAD23B0202), and the funds of Shandong "Double Tops" program (SYL2017XTTD02. The funders had no role in study design, data collection and analysis, decision to publish, or preparation of the manuscript.

\section{Abstract}

Studying soil nutrient variability and its effect on the growth and development of crops under a traditional tillage mode is the foundation for comprehensively implementing precision agriculture policies at the field scale and ensuring excellent crop management. In this paper, a $28.5 \mathrm{hm}^{2}$ winter wheat field under the traditional cultivation model in Tianzhuang town of Huantai County was selected as the research area. The mesh point method was utilized for sampling $(60 \times 60 \mathrm{~m})$, and the characteristics of soil available nitrogen (AN), available phosphorus (AP) and available potassium (AK) variations in the before sowing, reviving, jointing, and filling stages of winter wheat were analyzed using geostatistical and GIS methods. Moreover, Pearson correlation analysis was used to study the response of wheat growth and development to soil nutrient variations. As the growth stages progressed, 1) each nutrient showed the characteristics of low-high-low and moderate variability. The highest AN and AK contents were found at the reviving stage, while AP reached a turning point at the jointing stage. The order of variability of each nutrient was $A N>A P>A K .2)$ The nutrient variations first increased and then decreased and showed medium to strong spatial correlation. The three nutrients were strongly spatially correlated in the before sowing stage and moderately spatially correlated during the reviving stage. During the jointing and filling stages, AN had moderate spatial correlation, and AP and AK had strong spatial correlation. The spatial correlation of each nutrient was the weakest in the reviving stage, and the spatial correlation of AN was strongest in the before sowing stage, while the spatial correlations of $A P$ and AK were strongest in the jointing stage. The spatial correlation of each soil nutrient decreased from the before sowing stage to the reviving stage and from the jointing stage to the filling stage, and the spatial correlation increased from the reviving stage to the jointing stage. 3) The soil nutrient content first increased and then decreased, and the grades of the nutrients gradually decreased. 4) The correlation between soil nutrients and wheat growth gradually increased. AN had the highest correlation with wheat growth, followed by AK and AP. The effect of soil nutrients on the growth of wheat at the reviving stage was greater than the effect of nutrients in the current stage. The growth of wheat at the jointing stage was mainly influenced by nutrients in the current stage, while the growth of wheat at the filling stage was influenced by the nutrient contents of both the previous and current 
Competing interests: The authors have declared that no competing interests exist. stages. Thus, the date of fertilizer supplementation should be postponed properly. In this study, the soil nutrient dynamics and their influence on the growth of wheat during the winter wheat growth period under the traditional field model were well described, and these results could provide a theoretical basis for the precision management of soil nutrients in the northern winter wheat area where the planting environment and cultivation management are relatively uniform.

\section{Introduction}

Research on soil and crops under field conditions is a key step in advancing precision agriculture from theory to practice. Soil continuously varies in time and space and is the carrier and nutrient source for plant growth [1-3]. The basis for precision management and fertilization of farmland is the understanding of the temporal and spatial variability in soil nutrients and the crop responses in different growth stages in time [4-7].

Soil nutrient variability has long been a research hotspot for scholars throughout the world [8-10], and many experiments have been carried out on various nutrients and at different scales. For example, Duraisamy et al. [11] studied the spatial heterogeneity of soil $\mathrm{pH}$, organic carbon and available nutrients in the typical dry areas of India. Rosemary et al. [12] studied the spatial structure of topsoil at multiple scales and land use intensities. Paz et al. [13] explained that soil nutrients and trace elements exhibited spatial dependence even in very small areas (approximately 1.8 hectares). Yang et al. [14] compared the scale effects of soil available phosphorus (AP) and available potassium (AK) on spatial variability under different sampling scales. Blanchet et al. [15] analyzed the effects of different land use patterns, soil types, topographies and other factors on the spatial variability of soil K. However, in general, most studies have focused on the spatial variability of soil nutrients, with less consideration of the temporal variability and their effects on crop growth. Thus, the spatiotemporal variability of soil nutrients in different crop growth stages remains unclear [16-18].

Santillano-Cázares et al. [19] showed that the temporal variability of soil nutrients was much greater than its spatial variability. Qiao et al. [20] studied the characteristics of the temporal and spatial variations in soil in wheat fields that used drip irrigation for different numbers of years. However, these studies are still limited to the analysis of soil nutrients themselves, and the research on soil nutrient variability combined with crop development is still very scarce. Plant growth depends on the absorption of soil nutrients, so soil nutrient variability should also cause spatiotemporal variability in plant growth status [21-24]. An analysis of the variability in soil nutrients and its correlation with crop growth will help to clarify the spatiotemporal variability of the variables in a farmland system and have positive impacts on production [25-27]. Meanwhile, we found that previous studies in this area mostly used field experiment methods. Although these methods can obtain better test results by controlling the test conditions, the conclusions have great limitations in the practical application of agricultural extension because the conditions are different from the real field conditions.

Based on this, the characteristics of the variations in soil nutrients and their effects on wheat growth were analyzed by investigating the traditional cultivation management model for the main winter wheat production areas in China to determine the drawbacks of this model and provide a scientific basis for fertilization in different growth stages of wheat. 


\section{Materials and methods}

\section{Ethics statement}

The research area of this study is located in Tianzhuang town, Huantai County. All necessary permits for the described field study have been obtained from Huantai agricultural bureau. The land accessed is not privately owned or protected. The field study did not involve endangered or protected species. The study did not involve animal husbandry or experimentation.

\section{Study area}

The study was conducted in Huantai County, which has an area of $28.5 \mathrm{hm}^{2}$ and is located in north-central Shandong Province, China $\left(37^{\circ} 1.98^{\prime} \sim 37^{\circ} 2.28^{\prime} \mathrm{N}, 117^{\circ} 59.7^{\prime} \sim 118^{\circ} 0.12^{\prime} \mathrm{E}\right)$. The study area is located in the Huanghuai winter wheat region, which is the most important wheat-producing area in China. The region was classified as having a continental monsoon climate in a warm temperate zone. The climate is mild, and the four seasons are distinct. The average annual air temperature is $12.5^{\circ} \mathrm{C}$, and the frost-free period lasts 197 days. The annual average precipitation is $587 \mathrm{~mm}$, which mainly falls in 6 9 months. The terrain is flat with an elevation of 5.7 to $6.8 \mathrm{~m}$ a.s.l. The main soil types are cinnamon soil (typic udivitrands), and the winter wheat variety is Jimai 17 . The entire process followed the traditional cultivation management mode of the farmers. The main points of the process are as follows: traditional plowing mode was adopted, and the plow depth was $20 \mathrm{~cm}$; basal compound fertilizer $[\mathrm{N}+$ $\mathrm{P}_{2} \mathrm{O}_{5}+\mathrm{K}_{2} \mathrm{O}<45 \%$ (15-15-15)] was applied before sowing on October 12, 2016, at a dosage of $360 \mathrm{~kg} / \mathrm{hm}^{2}$, sowing was planted by equal row spacing $(15 \mathrm{~cm})$, and the sowing depth was approximately $5 \mathrm{~cm}$; urea [CO $\left.\left(\mathrm{NH}_{2}\right)_{2}\right]$ was applied in the reviving stage on March 5, 2017, at a dosage of $300 \mathrm{~kg} / \mathrm{hm}^{2}$. The winter wheat was sowed on October 13, 2016, and harvested on June 10, 2017, for a total growth period of 260 days.

\section{Soil sampling and field survey}

First, we collected the basic land use status, soil and topographic maps for Huantai County and obtained the boundary of the study area. Second, a total of 81 sampling points were established according to the standard of uniform grid layout of $60 \mathrm{~m} \times 60 \mathrm{~m}$ (Fig 1). The coordinates of the sampling points were transformed and input into a Trimble Geo 7x series Global Position System (GPS), which has a positioning accuracy that can reach the centimeter level. Field surveys were conducted on October 8, 2016 (before sowing), March 10, 2017 (reviving stage), April 10, 2017 (jointing stage), and May 15, 2017 (filling stage).

Soil samples were collected by a five-point cross-sampling method with a sampling depth of 0-20 cm. Mixed soil samples from multiple points were brought back to the laboratory, dried, ground and sieved at $2 \mathrm{~mm}$. Soil available nitrogen (AN) was determined by the method of Cornfield [28]; available phosphorous was estimated by the method of Olsen, Cole [29]; available potassium was extracted by $1 \mathrm{~mol} / \mathrm{L}$ ammonium acetate $(\mathrm{pH}=7.0)$ and determined by the method of Schollenberger and Simon [30]. The chlorophyll contents (SPAD) of the wheat leaves were measured by a Minolta SPAD-502 chlorophyll content analyzer (Spectrum Technologies, Inc.). Five wheat plants were randomly selected at each observation point, and three measurements per plant were collected. The average value of the five wheat plants was calculated as the SPAD value for each sampling point. The leaf area index (LAI) of wheat was measured by a LAI-2200C (Li-Cor, Inc.) plant canopy analyzer, and each sampling point was measured by two methods: across the ridge and parallel to the ridge. Each measurement was collected three times. Finally, the mean value was considered as the LAI value for each point. 


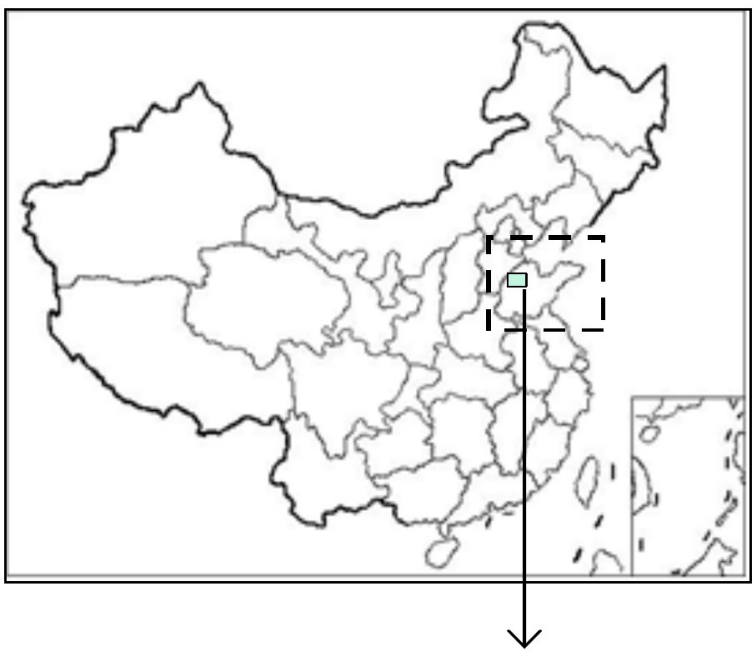

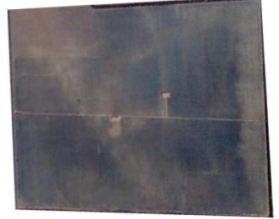

B

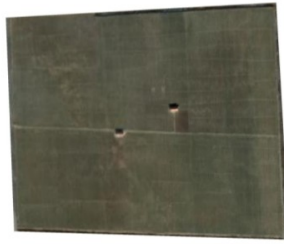

J

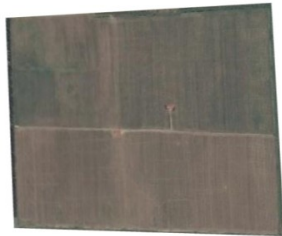

$\mathrm{R}$

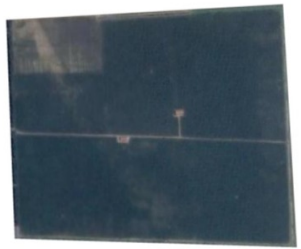

F
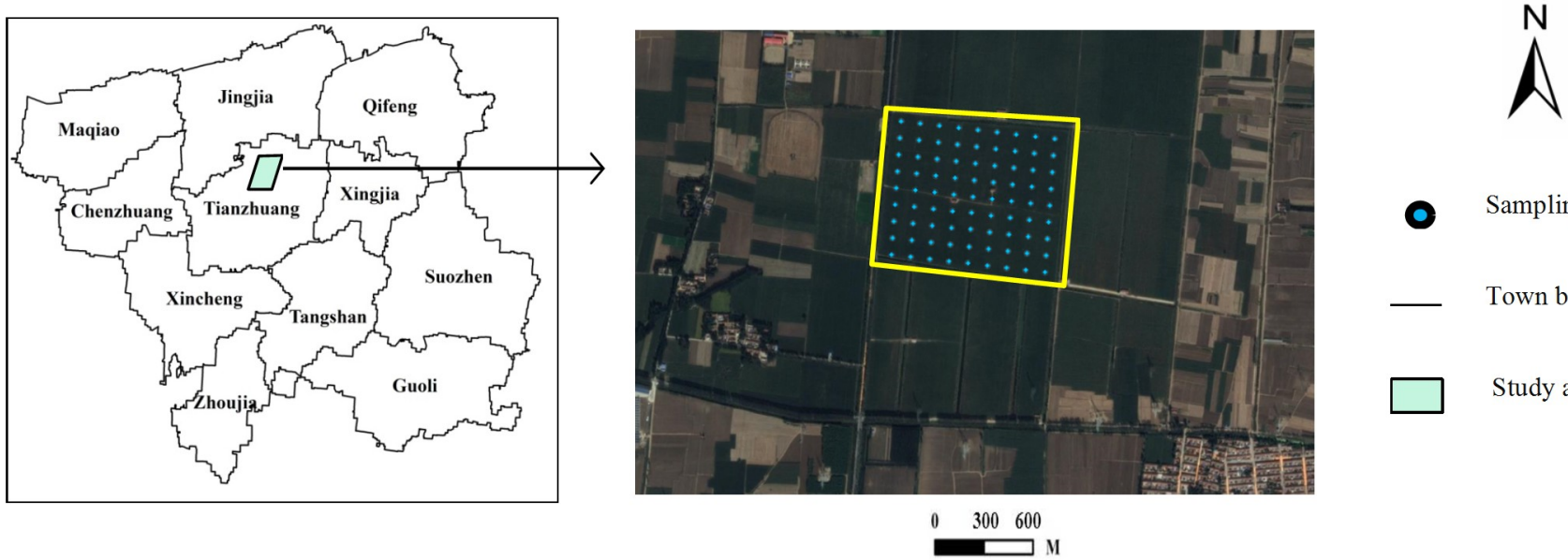

- Sampling points

Town boundary

Study area

Fig 1. Location of the study area and distribution of soil sampling sites. Note: B: before sowing; R: reviving stage; J: jointing stage; F: filling stage.

\section{Data processing and analysis methods}

Data processing. The interval method was used to select abnormal values. The interval is $[\mu-3 \mathrm{~s}, \mu+3 \mathrm{~s}], \mu$ is the mean value of the sample data, $\mathrm{s}$ is the standard deviation, and the outliers outside the interval are replaced by the normal maximum and minimum values.

Analysis methods. The eigenvalues of the classical statistical methods include minimum (min), maximum (max), mean, standard deviation (SD), skewness (Skew), kurtosis (Kurt) and coefficient of variation (CV), which can be used to describe the basic physical characteristics of soil nutrients. Among them, the $\mathrm{CV}$ is a parameter that can be used to compare the degree of data discretization under different measurement scales. According to the CV classification level, $<10 \%$ indicates weakly variability, $10 \%$ to $100 \%$ indicates medium variability, and $>100 \%$ indicates strong variability [31]. The single factor analysis of variance-least significant difference (LSD) method in SPSS software was used to analyze the significant differences in soil nutrients in different growth stages, and the level of confidence was 0.05 .

The spatiotemporal variability of soil nutrients was mainly analyzed by a semivariogram. Moran's I and fractal dimension (D) were combined to determine the spatial correlation and 
agglomeration degree at the same time. The formula for Moran's I is as follows:

$$
I=\frac{n}{\sum_{i} \sum_{j} \omega_{i, j}} \frac{\sum_{i} \sum_{j} \omega_{i, j}\left(x_{i}-\bar{x}\right)\left(x_{j}-\bar{x}\right)}{\sum_{i}\left(x_{i}-\bar{x}\right)^{2}}
$$

When calculating the value of Moran's I, it usually gives a threshold that can determine whether to reject the null hypothesis, called $Z$. If the value of $Z$ is in the range of -1.96-1.96, then $P>0.05$, indicating that null hypothesis of spatial randomness cannot be rejected; If the value of $Z$ is out of this range $(Z>1.96$ or $Z<-1.96)$, then the spatial pattern cannot be the result of random process. In this case, the null hypothesis can be rejected, and the spatial distribution shown is a significant aggregation or dispersion pattern [32]. The value of Moran's I is between -1 and 1 . When the adjacent region has a similar attribute value, the value of Moran's I is positive. When the adjacent area is different, Moran's I is negative; when the attribute value exhibits pure randomness, Moran's I tends to be 0 . First, the AN, AP and AK data for each growth stage were input to Gs+ software, and the distribution point maps were obtained by a semivariogram. Then, the point maps were fitted with spherical, exponential, Gaussian and linear models. Finally, the best model and its corresponding parameter index were obtained. Among the results, the values of $\mathrm{C}_{0} /\left(\mathrm{C}_{0}+\mathrm{C}\right)$ represent the proportion of the variation caused by random factors to the total spatial variation and are often used as the basis of the classification of the spatial variation in variables. When $\mathrm{C}_{0} /\left(\mathrm{C}_{0}+\mathrm{C}\right)<25 \%$, the system has a strong spatial correlation; when it is between $25 \%$ and $75 \%$, the system has a moderate spatial correlation; when it is $>75 \%$, the system has a weak spatial correlation [33].

The spatial distribution map of soil AN, AP and AK in different growth stages was plotted using the ordinary kriging interpolation method. The classification standard for soil nutrients in the interpolation map was formulated according to China's second national soil survey. AN was divided into 3 categories and 6 grades: high: I, $>150 \mathrm{mg} / \mathrm{kg}$, II, $120-150 \mathrm{mg} / \mathrm{kg}$; medium: III, 90-120 mg/kg, IV, 60-90 mg/kg; low: V, 30-60 mg/kg, VI, <30 mg/kg. AP was divided into 3 categories and 7 grades: high: I, $>100 \mathrm{mg} / \mathrm{kg}$, II, $60-100 \mathrm{mg} / \mathrm{kg}$; medium: III, $40-60 \mathrm{mg} /$ $\mathrm{kg}$, IV, 20-40 mg/kg, V, 10-20 mg/kg; low: VI, 5-10 mg/kg, VII, <5 mg/kg. AK was divided into 3 categories and 7 grades: high: I, $>400 \mathrm{mg} / \mathrm{kg}$, II, 350-400 mg/kg; medium: III, 300-350 $\mathrm{mg} / \mathrm{kg}, \mathrm{IV}, 250-300 \mathrm{mg} / \mathrm{kg}, \mathrm{V}, 200-250 \mathrm{mg} / \mathrm{kg}$; low: VI, 150-200 mg/kg, VII, <150 mg/kg. Based on the interpolated nutrient map, the professional viewpoints of landscape ecology were referenced, including 2 landscape level indices: patch number (NP) and patch density (PD) and 1 type level index: the percentage of landscape area (PLAND). A total of 3 indices were selected to quantitatively analyze the spatial dynamic characteristics of soil nutrient variation.

The values of SPAD and LAI at different growth stage of wheat and the soil AN, AP, and AK were compared at each point. The correlation coefficient was calculated using Pearson correlation analysis to analyze the response of winter wheat growth to soil nutrient changes.

\section{Results and analysis}

\section{Descriptive statistical analysis of soil nutrients in different growth stages of winter wheat}

The statistical characteristics of soil nutrients in different growth stages of winter wheat are shown in Table 1. The AN content ranged from 50.82 to $91.24 \mathrm{mg} / \mathrm{kg}$, and the CV ranged from $51.50 \%$ to $56.47 \%$ from before the sowing stage to the filling stage. The AN content reached a turning point at the reviving stage. The AN content increased slightly before the reviving stage and then decreased significantly after the reviving stage. There was no significant difference in AN content between the before sowing stage and reviving stages, the jointing 
Table 1. Statistical characteristics of soil nutrients during the different growth stages of winter wheat.

\begin{tabular}{|c|c|c|c|c|c|c|c|c|c|c|c|}
\hline Index & Growth stage & $\operatorname{Min}(\mathrm{mg} / \mathrm{kg})$ & $\operatorname{Max}(\mathrm{mg} / \mathrm{kg})$ & Mean (mg/kg) & $\mathrm{SD}(\mathrm{mg} / \mathrm{kg})$ & Skew & Kurt & K-S test & CV (\%) & Significance & $\mathbf{F}$ \\
\hline \multirow[t]{4}{*}{$\mathrm{AN}$} & B & 15.29 & 213.27 & $88.73 \mathrm{a}$ & 45.06 & 0.66 & 0.01 & 0.42 & 51.50 & 0.00 & $22.01^{* *}$ \\
\hline & $\mathrm{R}$ & 13.83 & 259.25 & $91.24 \mathrm{a}$ & 55.71 & 0.71 & 0.25 & 0.37 & 56.47 & & \\
\hline & $\mathrm{J}$ & 3.82 & 116.95 & $54.45 \mathrm{~b}$ & 28.09 & 0.37 & -0.72 & 0.56 & 51.59 & & \\
\hline & $\mathrm{F}$ & 8.35 & 99.54 & $50.82 \mathrm{~b}$ & 26.75 & 0.10 & -1.25 & 0.21 & 55.89 & & \\
\hline \multirow[t]{4}{*}{$\mathrm{AP}$} & B & 4.90 & 95.28 & $35.70 \mathrm{~b}$ & 20.39 & 0.96 & 0.93 & 0.47 & 59.14 & 0.00 & $24.91^{* *}$ \\
\hline & $\mathrm{R}$ & 16.94 & 102.48 & $59.49 \mathrm{a}$ & 20.56 & 0.29 & -0.50 & 0.57 & 34.56 & & \\
\hline & $\mathrm{J}$ & 15.32 & 133.85 & $64.37 \mathrm{a}$ & 28.11 & 0.50 & -0.38 & 0.66 & 44.53 & & \\
\hline & $\mathrm{F}$ & 16.70 & 109.70 & $57.37 \mathrm{a}$ & 19.62 & 0.62 & 0.59 & 0.36 & 34.95 & & \\
\hline \multirow[t]{4}{*}{$\mathrm{AK}$} & B & 125.79 & 474.4 & $285.19 \mathrm{~b}$ & 80.02 & 0.07 & -0.86 & 0.10 & 28.06 & 0.00 & $26.74^{* *}$ \\
\hline & $\mathrm{R}$ & 197.47 & 408.41 & $377.13 \mathrm{a}$ & 95.62 & 0.31 & -0.24 & 0.95 & 25.22 & & \\
\hline & $\mathrm{J}$ & 170.11 & 406.14 & $299.52 \mathrm{~b}$ & 50.43 & -0.29 & 0.16 & 0.20 & 16.84 & & \\
\hline & $\mathrm{F}$ & 181.23 & 412.15 & 292.08 b & 70.32 & 0.07 & -1.21 & 0.57 & 24.08 & & \\
\hline
\end{tabular}

Note: AN: available N; AP: available P; AK: available K. B: before sowing; R: reviving stage; J: jointing stage; F: filling stage. The same is true below. The LSD method was used for multiple comparisons. The same letters in each column indicate that there were no significant differences in the average value. The difference was significant when the significance was $<0.05$,

**extremely significant.

and filling stages, but the AN content in the before sowing and reviving stages was significantly higher than that during the jointing and filling stages. The AP content ranged from 35.70 to $64.37 \mathrm{mg} / \mathrm{kg}$, and the CV ranged from $34.56 \%$ to $59.14 \%$ from the before sowing stage to the filling stage. The AP content was low before sowing and was significantly different from that in the other three growth stages. At the reviving stage, the AP content increased significantly and increased slightly at the jointing stage and then decreased slightly at the filling stage. The $\mathrm{CV}$ for AP content was moderate in each growth stage, but the CV before sowing appeared higher, followed by jointing stage, and the lowest values occurred at the reviving stage and filling stage. The AK content ranged from 285.19 to $377.13 \mathrm{mg} / \mathrm{kg}$, and the $\mathrm{CV}$ ranged from $16.84 \%$ to $28.06 \%$ from the before sowing stage to the filling stage. The AK content in the reviving stage was significantly higher than that in the other stages. The AK content increased significantly from the before sowing stage to the reviving stage but decreased significantly at the jointing stage. The $\mathrm{CV}$ for $\mathrm{AK}$ was moderate in each growth stage, slightly higher before sowing, lower at the jointing stage, and moderate at the reviving stage and filling stage, but the differences were not significant.

In general, the soil nutrient contents showed the characteristics of low-high-low and moderate variability at the different growth stages. The AN and AK contents were highest in the reviving stage and $\mathrm{AP}$ was in the jointing stage. The $\mathrm{AN}$ content in the study area was relatively low, while the AP and AK contents were high according to the grading standard of China's second national soil survey. The order of the degree of variation in each nutrient was $\mathrm{AN}>\mathrm{AP}>\mathrm{AK}$. The results of the Kolmogorov-Smirnov test showed that under the $5 \%$ test standard, the AN, AP and AK of each growth stage obeyed a normal distribution after logarithmic transformation, which met the requirements for Moran's I and kriging interpolation.

\section{The characteristics of soil nutrient variations in different growth stages of winter wheat}

The semivariogram models and parameters for soil nutrients in different growth stages of winter wheat are shown in S1 Fig, Table 2. The best-fitting models for the three nutrients before 
Table 2. Semivariogram theoretical models and parameters of soil nutrients during the different growth stages of winter wheat.

\begin{tabular}{|c|c|c|c|c|c|c|c|c|c|c|c|}
\hline Growth stage & Index & Model & $\mathrm{C}_{0}$ & $\mathrm{C}_{0}+\mathrm{C}$ & $\mathrm{C}_{0} /\left(\mathrm{C}_{0}+\mathrm{C}\right)(\%)$ & $A(\mathbf{m})$ & $\mathbf{R}^{2}$ & RMSSE & D & $I$ & $\mathbf{Z}$ \\
\hline \multirow[t]{3}{*}{ B } & AN & E & 0.0060 & 0.0583 & 10.29 & 245.7 & 0.941 & $1.68 \times 10^{-5}$ & 1.857 & 0.305 & 2.65 \\
\hline & $\mathrm{AP}$ & E & 0.0073 & 0.0844 & 8.65 & 164.7 & 0.993 & $2.13 \times 10^{-6}$ & 1.897 & 0.226 & 3.42 \\
\hline & $\mathrm{AK}$ & $\mathrm{E}$ & 0.0024 & 0.0192 & 12.5 & 235.2 & 0.976 & $7.79 \times 10^{-7}$ & 1.817 & 0.320 & 2.22 \\
\hline \multirow[t]{3}{*}{$\mathrm{R}$} & AN & $\mathrm{E}$ & 0.0408 & 0.1055 & 38.67 & 179.8 & 0.969 & $2.10 \times 10^{-5}$ & 1.887 & 0.282 & 2.44 \\
\hline & $\mathrm{AP}$ & S & 0.0090 & 0.0262 & 34.35 & 142.5 & 0.920 & $3.08 \times 10^{-6}$ & 1.925 & 0.209 & 1.99 \\
\hline & $\mathrm{AK}$ & S & 0.0036 & 0.0138 & 26.09 & 235.8 & 0.997 & $8.70 \times 10^{-8}$ & 1.914 & 0.153 & 4.26 \\
\hline \multirow[t]{3}{*}{$\mathrm{J}$} & AN & $S$ & 0.0233 & 0.0860 & 27.09 & 146.8 & 0.913 & $4.92 \times 10^{-5}$ & 1.855 & 0.280 & 2.85 \\
\hline & $\mathrm{AP}$ & E & 0.0035 & 0.0419 & 8.35 & 122.7 & 0.907 & $5.49 \times 10^{-6}$ & 1.900 & 0.212 & 2.63 \\
\hline & $\mathrm{AK}$ & G & 0.0007 & 0.0065 & 10.77 & 108.1 & 0.982 & $3.36 \times 10^{-8}$ & 1.853 & 0.260 & 2.02 \\
\hline \multirow[t]{3}{*}{$\mathrm{F}$} & AN & G & 0.0333 & 0.0916 & 36.35 & 127.5 & 0.904 & $6.93 \times 10^{-5}$ & 1.890 & 0.270 & 1.98 \\
\hline & $\mathrm{AP}$ & G & 0.0048 & 0.0302 & 15.89 & 88.2 & 0.938 & $2.20 \times 10^{-6}$ & 1.941 & 0.177 & 2.03 \\
\hline & $\mathrm{AK}$ & $\mathrm{E}$ & 0.0017 & 0.0123 & 13.82 & 117.9 & 0.929 & $2.72 \times 10^{-7}$ & 1.934 & 0.125 & 2.26 \\
\hline
\end{tabular}

Note: E: exponential model; S: spherical model; G: Gaussian model. D: the fractal dimensions. I: Moran’s I.

sowing were all exponential models, indicating that each nutrient has a similar spatial variation trend and that the combination of the characteristics of soil nutrient variations was the simplest throughout the growth period. The best-fitting model for AN was the exponential model, while the best-fitting model for AP and AK was the spherical model at the reviving stage. The variation trends of AP and AK were similar but different from that of AN in this stage. The combination of the characteristics of soil nutrient variations was the most complicated in the jointing stage, which is contrary to that observed before sowing. The best-fitting models of $\mathrm{AN}, \mathrm{AP}$ and AK were spherical, exponential and Gaussian models, respectively. In the filling stage, the Gaussian model was best-fitting model for AN and AP, and the best-fitting model for AK was the exponential model, which showed that the spatial variation trends of AN and AP were similar. However, different from AK, the characteristics of the soil nutrient variations during the filling stage and the reviving stage were similar in complexity to those before sowing and during the jointing stage.

In general, the semivariogram of the three nutrients were represented by the same model at the before sowing stage, two models at the reviving stage, three models at the jointing stage, and then two models at the filling stage. The characteristics of the soil nutrient variations were greatest at the jointing stage, and the difference first increased and then decreased. The $\mathrm{R}^{2}$ of the best-fitting model for each nutrient ranged from 0.904 to 0.997 , and the root mean square standardized error (RMSSE) ranged from $3.36 \times 10^{-8}$ to $6.93 \times 10^{-5}$, which indicated that the selected model of the semivariogram was optimal and could adequately reflect the characteristics of the spatial structure and variation trends of soil nutrients throughout the growth period.

The $\mathrm{C}_{0}$ values of $\mathrm{AN}, \mathrm{AP}$ and $\mathrm{AK}$ in the four growth stages were all close to 0 , indicating that the variation caused by the test and sampling errors was very small. $\mathrm{The}_{0} /\left(\mathrm{C}_{0}+\mathrm{C}\right)$ of AN was $10.29 \%$ before sowing with strong spatial correlation, and it ranged from $27.09 \%$ to $38.67 \%$ from the reviving stage to the filling stage, with moderate spatial correlation. The $\mathrm{C}_{0} /$ $\left(\mathrm{C}_{0}+\mathrm{C}\right)$ values of $\mathrm{AP}$ at the before sowing, jointing and filling stages were $8.65 \%, 8.35 \%$ and $15.89 \%$, respectively, with strong spatial correlation, and this value was $34.35 \%$ at the reviving stage with moderate spatial correlation. The $\mathrm{C}_{0} /\left(\mathrm{C}_{0}+\mathrm{C}\right)$ values of $\mathrm{AK}$ at the before sowing, jointing and filling stages were $12.5 \%, 10.77$ and $13.82 \%$, respectively, with strong spatially correlated, and this value was $26.09 \%$ at the reviving stage with moderate spatial correlation. Overall, the three nutrients showed moderate to strong spatial correlation with the progression 
of the growth stages and experienced a process of weakening-strengthening-weakening. These three nutrients were strongly spatially correlated before sowing, and the spatial correlation was moderate during the reviving stage. For the jointing and filling stages, AN exhibited moderate spatial correlation, and AP and AK were strongly spatially correlated. These three nutrients exhibited the weakest spatial correlation at the reviving stage, the spatial correlation of AN was strongest in the before sowing stage, while the spatial correlations of AP and AK were the strongest at the jointing stage.

The fractal dimensions of the three nutrients were from 1.817-1.897, 1.887-1.925, 1.8531.900 and 1.890-1.941 from the before sowing stage to the filling stage, indicating that the soil nutrients in each growth stage had good fractal characteristics and structure. The fractal dimension values showed a process of first increasing then decreasing and then increasing from the before sowing stage. This result indicated that the structure of the soil nutrients became more complex, and the spatial correlation was weakened from the before sowing stage to the reviving stage and from the jointing stage to the filling stage, while the structure of nutrients tended to be consistent, and the spatial correlation increased from the reviving stage to the jointing stage.

The $\mathrm{Z}$ values of $\mathrm{AN}, \mathrm{AP}$ and $\mathrm{AK}$ in the four growth stages were all $>1.96$, indicated that the null hypothesis of spatial randomness could be rejected and the spatial distribution was a significant aggregation pattern. The spatial autocorrelation are shown in S2 Fig. Moran's I of the three nutrients ranged from $0.226-0.320,0.153-0.282,0.212-0.280$ and $0.125-0.270$ from the before sowing stage to the filling stage. The value of Moran's I showed a process of first decreasing then increasing and then decreasing from the before sowing stage. The results showed that the soil nutrient structure gradually dispersed, and the spatial correlation decreased from the before sowing stage to the reviving stage and from the jointing stage to the filling stage. The structure of the nutrients tended to gather, and the spatial correlation increased from the reviving stage to the filling stage.

In general, as the growth progressed, (1) each nutrient showed moderate to strong spatial correlation. (2) The three nutrients exhibited strong spatial correlation during the before sowing stage and moderate spatial correlation during the reviving stage. For the jointing and filling stages, AN exhibited a moderate spatial correlation, and AP and AK exhibited strong spatial correlation. (3) The weakest spatial correlation of the three nutrients was shown at the reviving stage; the strongest spatial correlation of AN was shown before sowing, while the AP and AK exhibited the strongest spatial correlation at the jointing stage. (4) The spatial correlation of soil nutrients decreased from before sowing to the reviving stage, jointing stage and filling stage, and increased from the reviving stage to the jointing stage.

\section{The spatial distribution characteristics of soil nutrients in different growth stages of winter wheat}

The spatial and temporal distribution maps of soil nutrients in the different growth stages of winter wheat are shown in Fig 2. The distribution of AN before sowing showed a striped pattern, and the content from northwest to southeast was obviously higher than that in other regions. For the reviving stage, the concentration of AN declined slightly, and the content in the study area was opposite to that before sowing. The areas with high AN contents were concentrated in the northeast to southwest areas. For the jointing stage, AN showed a clustered distribution, and the AN content was significantly decreased, mainly concentrated from 60 90 $\mathrm{mg} / \mathrm{kg}$. For the filling stage, the continuity of the distribution continued to increase, and the AN content continued to decline, reaching a value lower than $60 \mathrm{mg} / \mathrm{kg}$. The distribution of AP before sowing was dispersed, and there were no clear centers of high and low contents. For 


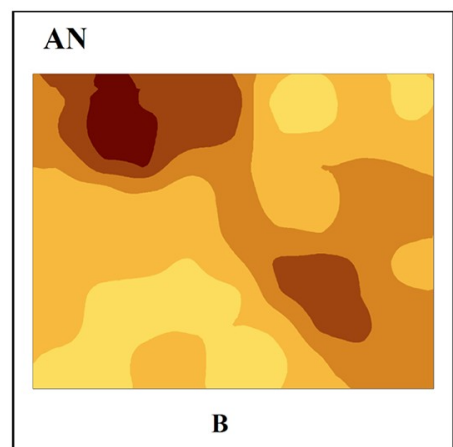

$\Lambda$

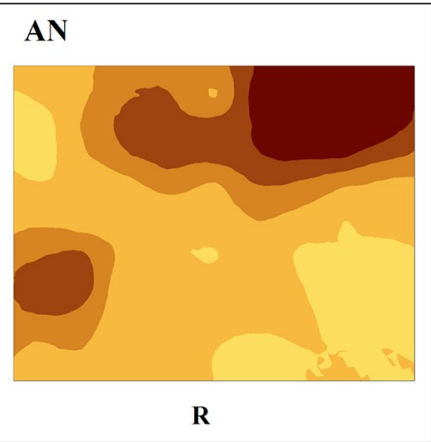

$\mathbf{0}$

0

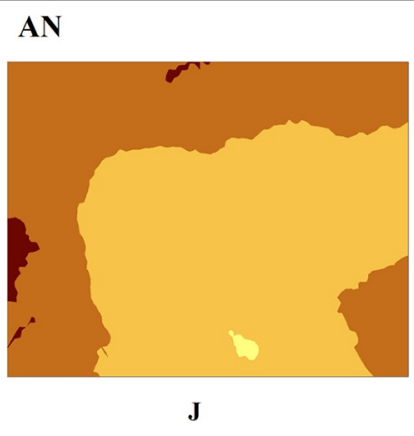

$\mathrm{AN}(\mathbf{m g} / \mathbf{k g})$
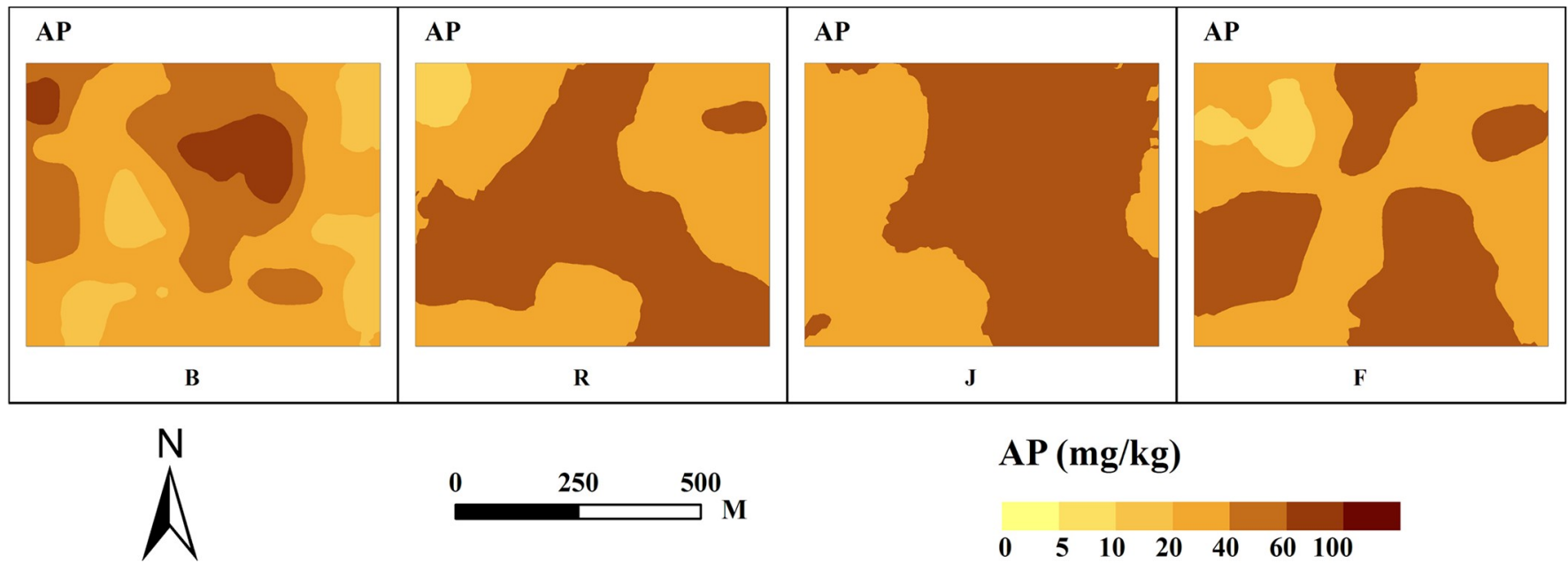

\section{AP (mg/kg)}

$\begin{array}{lllllll}0 & 5 & 10 & 20 & 40 & 60 & 100\end{array}$
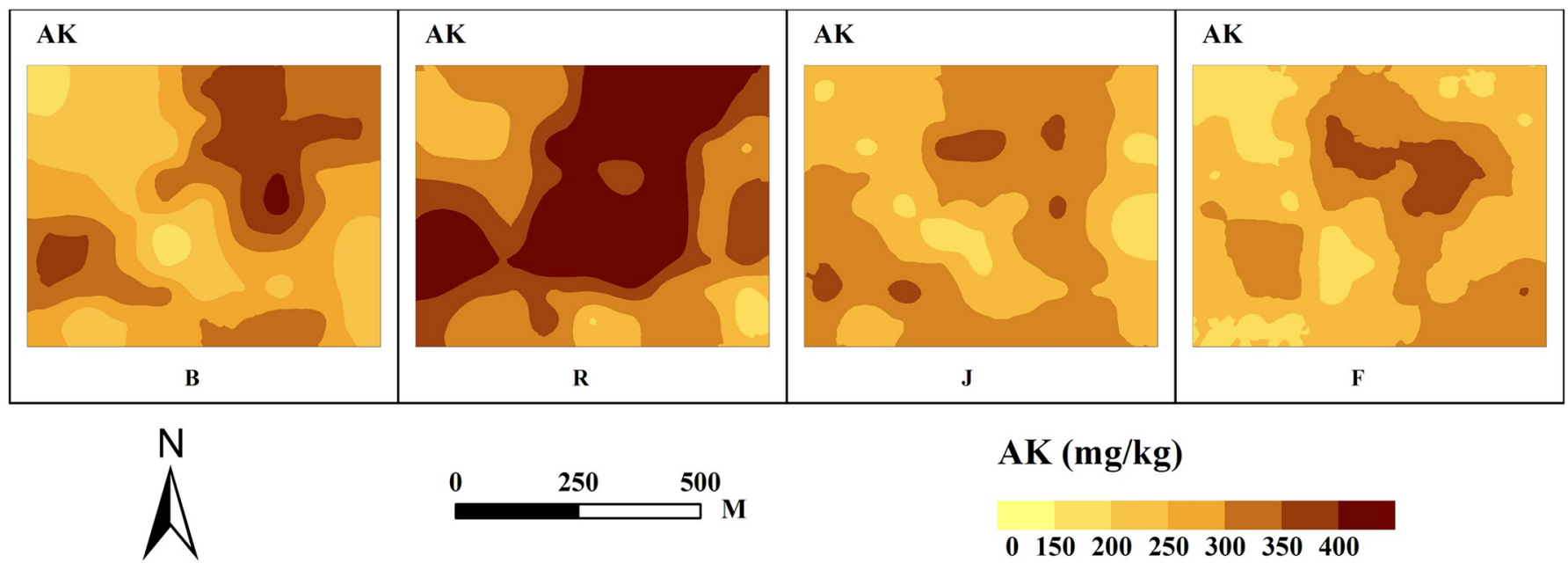

250

500

$\operatorname{AK}(\mathbf{m g} / \mathbf{k g})$

$\begin{array}{lllllll}0 & 150 & 200 & 250 & 300 & 350 & 400\end{array}$

Fig 2. Spatial distribution of soil nutrients during the different growth stages of winter wheat. Note: AN: available N; AP: available P; AK: available K. B: before sowing; R: reviving stage; J: jointing stage; F: filling stage.

https://doi.org/10.1371/journal.pone.0203509.g002 
Table 3. Variation in AN landscape indices at the landscape and class level during the different growth stages of winter wheat.

\begin{tabular}{|c|c|c|c|c|c|c|c|}
\hline Growth stage & Index & Class I & Class II & Class III & Class IV & Class V & Class VI \\
\hline \multirow[t]{3}{*}{ B } & NP & 1 & 2 & 1 & 5 & 3 & 1 \\
\hline & $\mathrm{PD}\left(/ \mathrm{hm}^{2}\right)$ & 0.0286 & 0.0571 & 0.0286 & 0.1429 & 0.0857 & 0.0286 \\
\hline & PLAND & $4.52 \%$ & $15.87 \%$ & $18.56 \%$ & $37.23 \%$ & $22.87 \%$ & $0.95 \%$ \\
\hline \multirow[t]{3}{*}{$\mathrm{R}$} & NP & 1 & 2 & 2 & 6 & 3 & \\
\hline & $\mathrm{PD}\left(/ \mathrm{hm}^{2}\right)$ & 0.0286 & 0.0571 & 0.0571 & 0.1714 & 0.0857 & \\
\hline & PLAND & $14.38 \%$ & $13.94 \%$ & $16.55 \%$ & $34.01 \%$ & $21.11 \%$ & \\
\hline \multirow[t]{3}{*}{$\mathrm{J}$} & NP & & & 5 & 2 & 1 & 1 \\
\hline & $\mathrm{PD}(/ \mathrm{hm} 2)$ & & & 0.1429 & 0.0571 & 0.0286 & 0.0286 \\
\hline & PLAND & & & $1.76 \%$ & $47.57 \%$ & $50.23 \%$ & $0.43 \%$ \\
\hline \multirow[t]{3}{*}{ F } & NP & & & & 2 & 4 & 2 \\
\hline & $\mathrm{PD}(/ \mathrm{hm} 2)$ & & & & 0.0571 & 0.1143 & 0.0571 \\
\hline & PLAND & & & & $27.60 \%$ & $71.27 \%$ & $1.13 \%$ \\
\hline
\end{tabular}

Note: NP: patch number; PD: patch density; PLAND: percentage of landscape area. The same is true below.

the reviving stage, the distribution of AP showed a striped pattern, and the AP content increased significantly at this stage. For the jointing stage, the boundaries of the high and low regions were obvious, the overall trend was high in the east and low in the west, and the AP content was high throughout the study area, mainly concentrated at $>60 \mathrm{mg} / \mathrm{kg}$. For the filling stage, AP showed a clustered distribution, and the area with high nutrient contents decreased. The distribution of AK before sowing showed a clustered distribution, and the high content area was concentrated in the northeast. For the reviving stage, the distribution of AK showed a striped pattern, and the area with high contents increased. For the jointing stage, the continuity of the AK distribution decreased significantly, and the area with high contents decreased rapidly. For the filling stage, the distribution characteristics of AK were similar to those at the jointing stage; the AK content decreased further, and the area with low contents increased.

The landscape pattern indices for the interpolation of soil nutrients in different growth stages of winter wheat are shown in Tables 3, 4 and 5. The distribution of AN was consistent at the landscape level during the different stages. Patch number and PD showed the characteristics of increased-decreased-decreased. From before sowing to the filling stage, the proportions

Table 4. Variation in AP landscape indices at the landscape and class level during the different growth stages of winter wheat.

\begin{tabular}{|c|c|c|c|c|c|c|c|c|}
\hline Growth stage & Index & Class I & Class II & Class III & Class IV & Class V & Class VI & Class VII \\
\hline \multirow[t]{3}{*}{$\mathrm{B}$} & NP & & 2 & 3 & 2 & 5 & 1 & 1 \\
\hline & $\mathrm{PD}\left(/ \mathrm{hm}^{2}\right)$ & & 0.0571 & 0.0857 & 0.0571 & 0.1429 & 0.0286 & 0.0286 \\
\hline & PLAND & & $10.18 \%$ & $31.96 \%$ & $39.11 \%$ & $17.21 \%$ & $0.81 \%$ & $0.72 \%$ \\
\hline \multirow[t]{3}{*}{$\mathrm{R}$} & $\mathrm{NP}$ & 1 & 3 & 3 & 1 & & & \\
\hline & $\mathrm{PD}\left(/ \mathrm{hm}^{2}\right)$ & 0.0286 & 0.0857 & 0.0857 & 0.0286 & & & \\
\hline & PLAND & $0.92 \%$ & $54.31 \%$ & $41.38 \%$ & $3.39 \%$ & & & \\
\hline \multirow[t]{3}{*}{$\mathrm{J}$} & NP & & 2 & 3 & & & & \\
\hline & $\mathrm{PD}\left(/ \mathrm{hm}^{2}\right)$ & & 0.0571 & 0.0857 & & & & \\
\hline & PLAND & & $67.81 \%$ & $32.17 \%$ & & & & \\
\hline \multirow[t]{3}{*}{$\mathrm{F}$} & NP & & 4 & 1 & 1 & & & \\
\hline & $\mathrm{PD}\left(/ \mathrm{hm}^{2}\right)$ & & 0.1143 & 0.0286 & 0.0286 & & & \\
\hline & PLAND & & $48.77 \%$ & $45.35 \%$ & $5.88 \%$ & & & \\
\hline
\end{tabular}

https://doi.org/10.1371/journal.pone.0203509.t004 
Table 5. Variation in AK landscape indices at the landscape and class level during the different growth stages of winter wheat.

\begin{tabular}{|c|c|c|c|c|c|c|c|c|}
\hline Growth stage & Index & Class I & Class II & Class III & Class IV & Class V & Class VI & Class VII \\
\hline \multirow[t]{3}{*}{ B } & NP & 1 & 2 & 3 & 1 & 4 & 2 & 1 \\
\hline & $\mathrm{PD}\left(/ \mathrm{hm}^{2}\right)$ & 0.0286 & 0.0571 & 0.0857 & 0.0286 & 0.1143 & 0.0571 & 0.0286 \\
\hline & PLAND & $1.83 \%$ & $16.30 \%$ & $21.99 \%$ & $26.13 \%$ & $28.28 \%$ & $3.95 \%$ & $1.51 \%$ \\
\hline \multirow[t]{3}{*}{$\mathrm{R}$} & NP & 2 & 3 & 3 & 4 & 2 & & \\
\hline & $\mathrm{PD}\left(/ \mathrm{hm}^{2}\right)$ & 0.0571 & 0.0857 & 0.0857 & 0.1143 & 0.0571 & & \\
\hline & PLAND & $33.87 \%$ & $21.72 \%$ & $30.19 \%$ & $12.86 \%$ & $1.36 \%$ & & \\
\hline \multirow[t]{3}{*}{$\mathrm{J}$} & NP & 1 & 5 & 1 & 3 & 6 & & \\
\hline & $\mathrm{PD}\left(/ \mathrm{hm}^{2}\right)$ & 0.0286 & 0.1429 & 0.0286 & 0.0857 & 0.1714 & & \\
\hline & PLAND & $1.24 \%$ & $5.14 \%$ & $55.85 \%$ & $31.46 \%$ & $6.31 \%$ & & \\
\hline \multirow[t]{3}{*}{$\mathrm{F}$} & NP & & 2 & 2 & 7 & 9 & 1 & \\
\hline & $\mathrm{PD}\left(/ \mathrm{hm}^{2}\right)$ & & 0.0571 & 0.0571 & 0.2 & 0.2571 & 0.0286 & \\
\hline & PLAND & & $6.34 \%$ & $31.16 \%$ & $47.27 \%$ & $14.13 \%$ & $1.10 \%$ & \\
\hline
\end{tabular}

of the areas that showed high grades (I and II) were $20.39 \%, 28.32 \%, 0 \%$, and $0 \%$, the proportions of the areas that showed middle graded (III and IV) were $55.79 \%, 50.56 \%, 49.33 \%$, and $27.6 \%$, and the proportions of the areas that showed low graded (V and VI) were $23.82 \%$, $21.11 \%, 50.66 \%$, and $72.4 \%$. This result showed that with the progression of the growth stages, the proportions of the area with high and low levels of AN exhibited an opposite trend, and the proportion of the area with high AN content gradually decreased after the reviving stage, while the proportion of the area with low grades increased. The distribution of AP was consistent at the landscape level during the different stages. Patch number and PD showed decreaseddecreased-increased characteristics. From before sowing to the filling stage, the proportions of the area with high grades (I and II) were $10.18 \%, 55.23 \%, 67.81 \%$, and $48.77 \%$, the proportions of the area with middle grades (III, IV, V) were $88.28 \%, 44.77 \%, 32.17 \%$, and $51.23 \%$, and the proportions of the areas with low grades (VI and VII) were $1.53 \%, 0 \%, 0 \%$, and $0 \%$. This result showed that the dominant areas were converted from high contents to moderate contents. The distribution of AK was consistent at the landscape level during the different stages and showed a characteristic of continuous increase. From before sowing to the filling stage, the proportions of the area with high grades (I and II) were $18.13 \%, 55.59 \%, 6.38 \%$, and $6.34 \%$, the proportions of the area with middle grades (III, IV, V) were $76.4 \%, 44.41 \%, 93.62 \%$, and $92.56 \%$, and the proportions of the area with low grades (VI and VII) were $5.46 \%, 0 \%, 0 \%$, and $1.1 \%$. This result showed that with the progression of the growth stages, the $\mathrm{AK}$ at the type level exhibited changes that were similar to the changes in AN in general, and the proportion of the area with high grades decreased after the reviving stage. Generally, throughout the growth stages of winter wheat, the contents of soil nutrients first increased and then gradually decreased, while the number of nutrient grades, especially the number of high content grades, gradually decreased.

\section{Correlation between soil nutrients and wheat growth during the same growth stage and subsequent stage}

The correlation coefficients between soil nutrients and the index of wheat growth are shown in Table 6. There were good correlations between the LAI of wheat and AN, AP, AK and SPAD. Among them, SPAD, LAI and AN reached significant positive correlations at each growth stage. There was not a significant correlation between SPAD and AP at any growth stage. There was a significant correlation between LAI and AP at the filling stage, but there was no correlation during the period from the reviving stage to the jointing stage. There was a significant correlation between SPAD and $\mathrm{AK}$ at the filling stage, but there was no correlation during 
Table 6. Correlation coefficients between soil nutrients and wheat growth stage.

\begin{tabular}{l|c|c|c|c}
\hline Growth stage & Index & AN & AP & AK \\
\hline \multirow{2}{*}{$\mathrm{R}$} & SPAD & $0.2823^{*}$ & 0.146 & 0.1683 \\
\cline { 2 - 5 } & LAI & $0.2097^{*}$ & 0.1147 & 0.1598 \\
\hline \multirow{2}{*}{$\mathrm{F}$} & SPAD & $0.5007^{* *}$ & 0.1737 & $0.2063^{*}$ \\
\cline { 2 - 5 } & LAI & $0.5412^{* *}$ & 0.1956 & $0.2587^{*}$ \\
\cline { 2 - 5 } & SPAD & $0.5168^{* *}$ & $0.2136^{*}$ & $0.265^{*}$ \\
\hline
\end{tabular}

Note:

${ }^{* *} \mathrm{P}<0.01$

${ }^{*} \mathrm{P}<0.05$. The same is true below.

the period from the reviving stage to the jointing stage. There was no correlation between LAI and $\mathrm{AK}$ at the filling stage, but it reached a significant correlation during the period from the reviving stage to the jointing stage.

With the increase or decrease in AN content in the soil, the SPAD and LAI values also increased or decreased correspondingly during the period from the reviving stage to the filling stage. With the increase in AP content in the soil, the LAI values also increased correspondingly at the filling stage, while the change in SPAD at the filling stage and the changes in SPAD and LAI at the other three stages were not obvious. These results showed that AP had different effects on wheat growth at different growth stages. The SPAD and LAI values were significantly correlated with the AK content in the soil during the jointing stage to the filling stage, and the correlation coefficients between these two growth stages were not significantly different. This result indicated that AK played an important role during the late growth stage of wheat and had a steady effect on wheat growth compared with AN and AP.

The correlation coefficients between the soil nutrient contents at the current stage and the index of wheat growth at the following growth stage are shown in Table 7. There was also a good correlation between the soil nutrient content at the current stage and the index of wheat growth at the following growth stage. There were extremely significant correlations between AN (before sowing) and SPAD (reviving stage), AN (jointing stage) and SPAD (filling stage),

Table 7. Correlation coefficients between soil nutrients and wheat growth stage of the following growth stage.

\begin{tabular}{|c|c|c|c|}
\hline Growth stage & Index & SPAD-2 & LAI-2 \\
\hline \multirow[t]{3}{*}{$\mathrm{R}$} & AN & $0.3204^{* *}$ & $0.2722^{*}$ \\
\hline & $\mathrm{AP}$ & 0.183 & 0.0964 \\
\hline & $\mathrm{AK}$ & $0.2049^{*}$ & 0.1874 \\
\hline \multirow[t]{3}{*}{$\mathrm{J}$} & AN & 0.1714 & 0.1241 \\
\hline & $\mathrm{AP}$ & 0.045 & 0.084 \\
\hline & $\mathrm{AK}$ & 0.1158 & 0.045 \\
\hline \multirow[t]{3}{*}{$\mathrm{F}$} & AN & $0.461^{* *}$ & $0.4922^{* *}$ \\
\hline & $\mathrm{AP}$ & 0.1804 & 0.1541 \\
\hline & $\mathrm{AK}$ & $0.2561^{*}$ & $0.2241^{*}$ \\
\hline
\end{tabular}

Note: $\mathrm{XX}-2$ is the index of winter wheat growth in the following stage;

${ }^{* *} \mathrm{P}<0.01$

${ }^{*} \mathrm{P}<0.05$.

https://doi.org/10.1371/journal.pone.0203509.t007 
LAI (filling stage). There were significant correlations between AN (before sowing) and LAI (reviving stage), AK (before sowing) and SPAD (reviving stage), AK (jointing stage) and SPAD, and AK (jointing stage) and LAI (filling stage).

The comparison of Tables 6 and 7 indicates that the correlation coefficients between AN, $\mathrm{AP}$ and $\mathrm{AK}$ (before sowing) and the SPAD and LAI (reviving stage) values were significantly higher than the correlation coefficients between AN, AP and AK (reviving stage) and the SPAD and LAI values (reviving stage). This result indicated that the nutrients before sowing had a more obvious effect on the growth of wheat at the reviving stage than the nutrients at the reviving stage. The correlation coefficients between AN, AP and AK (reviving stage) and the SPAD and LAI values (jointing stage) were significantly lower than the correlation coefficients between AN, AP and AK (jointing stage) and the SPAD and LAI values (jointing stage). This result indicated that the growth of wheat at the jointing stage was mainly affected by the nutrients of the current stage, which was related to the jointing stage being a peak period of nutrient uptake by wheat. This finding was mainly related to the reason that the jointing stage is a peak period of soil nutrient absorption by wheat. The correlation coefficients between AN, AP and AK (jointing stage) and the SPAD and LAI values (filling stage) were significantly lower than the correlation coefficients between AN, AP and AK (filling stage) and the SPAD and LAI values (filling stage), but it still reached significant correlation. This result indicated that the growth of wheat at the filling stage was affected by the soil nutrients at both the jointing stage and filling stage.

In general, the growth status of wheat was closely related to the nutrients in the soil. AN had the highest correlation with wheat growth status, followed by AK and AP. At the reviving stage, the effect of nutrients before sowing on the growth of wheat was slightly higher than the effect of nutrients in the reviving stage. At the jointing stage, the effect of nutrients in the jointing stage on the growth of wheat was much higher than the effect of nutrients in the reviving stage. At the filling stage, the status of wheat growth was affected by the nutrients in the jointing stage and filling stage.

\section{Discussion}

(1) Many experimental design methods have the advantages of easy access to data, low cost and small interference, but these conditions are different from the real field environment and management mode, and the conclusions of these studies have great limitations in the application of agricultural extension. Based on this, this research studied the soil nutrients and winter wheat under the traditional tillage model, and the conclusions were more practical and could be more suitable for winter wheat planting areas in northern China.

(2) Huantai County is located in the Huanghuai winter wheat area, which is the main wheat production area of China where the planting conditions are superior. It was representative to study the variation in soil nutrients and their correlation with crop growth during the winter wheat growth period. The minimum spatial correlation distance of soil nutrients in each growth stage was $88.2 \mathrm{~m}$, which exceeded the sampling interval $(60 \mathrm{~m})$ and indicated that the sampling interval was reasonable. In this study, the dynamic changes in soil nutrient content during the winter wheat growth period were generally consistent with the results of research conducted in Jiangyan City, Jiangsu Province, China [34].

(3) Soil nutrients showed moderate variations during the growth stage of winter wheat, generally showing that the concentrations of AN were slightly higher than those of AP but much larger than those of AK. The reason for this difference was mainly related to fertilization. The high variability of AN was due to the large demand for nitrogen by wheat, and farmers pay great attention to the use of urea. The high variability of AP was related to the chemical 
behavior of $\mathrm{P}$ elements in the soil [35]. The mobility of AP was weak, and its utilization rate was low, resulting in a large amount of fertilizer residue in the soil and uneven distribution, which was also confirmed by the maximum variation in AP before sowing in this research. The soil parent material in the study area was rich in potassium and was in excess of that required for the growth of wheat. Therefore, the influence of field management on AK was small, so the variability was low.

(4) Single $\mathrm{C}_{0} /\left(\mathrm{C}_{0}+\mathrm{C}\right)$ values were not stable or effective in describing the structure of the variation when the $\mathrm{C}_{0} /\left(\mathrm{C}_{0}+\mathrm{C}\right)$ values were large in a small area [36]. Therefore, a method of mutual verification of $\mathrm{C}_{0} /\left(\mathrm{C}_{0}+\mathrm{C}\right)$, fractal dimension and Moran's I was adopted in this research. The results showed that the structure of soil nutrients weakened from before sowing to the reviving stage and from the jointing stage to the filling stage and tended to strengthen from the reviving stage to the jointing stage. This pattern may be caused by field management and nutrient absorption during wheat growth. In particular, urea supplementation at the reviving stage and irrigation at the filling stage were the main reasons for the obvious decreases in soil nutrient correlation. The nutrient uptake of winter wheat peaked at the jointing stage, and the soil nutrient distribution tended to be balanced and then structurally enhanced. Significant changes in soil nutrient correlation were also found during the growth period of tobacco [37]. Wheat is one of the most important food crops in the world; thus, systematically studying the effects of wheat growth on soil nutrients was of great significance. However, the results in this field still need to be enriched.

(5) The correlation coefficients between AN and SPAD and LAI values were the highest at all growth stages of winter wheat, followed by those for AK and AP. This result may be related to the differences in the demands for different nutrients during the growth stage of winter wheat. The ratio of nitrogen, phosphorus and potassium absorption in wheat was 3.1:1:3.14 in the brown soil area of western Henan Province, China [38], which has planting conditions similar to those in this research area. This finding was basically consistent with the conclusions of this study. The correlation between the soil nutrients and the growth index of the following growth stage showed that the growth of the winter wheat in the reviving stage was mainly influenced by the soil nutrients in the previous stage (before sowing), indicating that there was a temporal delay from fertilization to the impacts of fertilizer on crop growth. The effects of soil nutrients at the jointing stage on the growth of wheat at the jointing stage were much higher than the effects of soil nutrients in the jointing stage. This result was related to the high demands for fertilizer at the jointing stage of wheat [39]. Therefore, ensuring the supply of fertilizer at the jointing stage is of great significance. At the filling stage, the effect of nutrients in the previous stage (jointing stage) on the growth of winter wheat was obvious. Because the traditional period of urea supplementation is generally during the reviving stage in northern China, in view of the importance of the jointing stage to the growth of winter wheat, the time of fertilizer supplementation should be properly moved to the jointing stage to ensure the nutrient supply in the later stage of wheat growth. The spatial and temporal distributions of more biological indicators of winter wheat need to be further studied.

\section{Conclusion}

(1) The contents of three nutrients all showed the characteristics of decreased-increaseddecreased and moderate variability. The AN content reached a turning point at the reviving stage, while the AP and AK contents reached turning points at the jointing stage. AN had the largest CV, followed by AP and AK.

(2) The three nutrients all showed medium to strong spatial correlation. The three nutrients showed the weakest spatial correlation at the reviving stage; the strongest spatial correlation of 
AN occurred before sowing, while the strongest spatial correlations of AP and AK were at the jointing stage. The correlation of soil nutrients decreased from before sowing to the reviving stage, from the jointing stage to the filling stage, and increased from the reviving stage to the jointing stage.

(3) The high AN contents gradually decreased, and the spatial distribution continuously increased; the high AP contents decreased slightly, the change in the spatial distribution continuity was not obvious; the high AK contents significantly decreased, and the spatial distribution continuity continuously decreased.

(4) AN exhibited the highest correlation with wheat growth status, followed by AK and AP. At the reviving stage, the effect of nutrients before sowing on the growth of wheat was slightly higher than that at the reviving stage. At the jointing stage, the effect of the nutrients in the jointing stage on the growth of wheat was much higher than that in the reviving stage. At the filling stage, the status of wheat growth was affected by the nutrients in both the jointing stage and filling stage.

The temporal and spatial characteristics of the soil nutrient variations and the response of wheat growth throughout the winter wheat growth period under the traditional tillage model were clearly described, which could provide a scientific basis for the effective guidance for wheat production and the popularization of precision fertilization technology.

\section{Supporting information}

S1 Fig. Semivariogram theoretical models of soil nutrients in different growth stages of winter wheat.

(DOCX)

S2 Fig. Spatial autocorrelation of soil nutrients in different growth stages of winter wheat. (DOCX)

\section{Acknowledgments}

We thank the agriculturist at the Agricultural Bureau of Huantai County for their help with the field work. We also thank the language expert from the American Journal Experts (AJE) language editing service for improving the language quality of this manuscript and the anonymous reviewers for reviewing this manuscript and providing comments.

\section{Author Contributions}

Conceptualization: Gengxing Zhao.

Formal analysis: Baowei Su.

Funding acquisition: Gengxing Zhao.

Investigation: Baowei Su, Chao Dong.

Methodology: Baowei Su, Gengxing Zhao.

Project administration: Gengxing Zhao.

Resources: Gengxing Zhao.

Software: Baowei Su, Chao Dong.

Writing - original draft: Baowei Su.

Writing - review \& editing: Baowei Su. 


\section{References}

1. Olmo M, Lozano AM, Barrón V, Villar R. Spatial heterogeneity of soil biochar content affects soil quality and wheat growth and yield. Sci Total Environ. 2016; 562:690-700. https://doi.org/10.1016/j.scitotenv. 2016.04.089 PMID: 27110980

2. Mathur M. Spatio-temporal variability in distribution patterns of Tribulus terrestris: linking patterns and processes. Journal of Agricultural Science \& Technology. 2014; 16:1187-201.

3. Boussadia O, Steppe K, Zgallai H, Hadj SBE, Braham M, Lemeur R, et al. Effects of nitrogen deficiency on leaf photosynthesis, carbohydrate status and biomass production in two olive cultivars 'Meski' and 'Koroneiki'. Sci Hortic-amsterdam. 2009; 123:336-42.

4. Marchal G, Smith KEC, Rein A, Winding A, Jonge LWd, Trapp S, et al. Impact of activated carbon, biochar and compost on the desorption and mineralization of phenanthrene in soil. Environ Pollut. 2013; 181:200-210. https://doi.org/10.1016/j.envpol.2013.06.026 PMID: 23871817

5. Maynard JJ, Johnson MG. Uncoupling the complexity of forest soil variation: Influence of terrain indices, spectral indices, and spatial variability. Forest Ecol Manag. 2016; 369:89-101.

6. Wang $\mathrm{T}$, Kang F, Cheng X, Han H, Bai Y, Ma J. Spatial variability of organic carbon and total nitrogen in the soils of a subalpine forested catchment at Mt. Taiyue, China. Catena. 2017; 155:41-52.

7. Zingore S, Murwira HK, Delve RJ, Giller KE. Influence of nutrient management strategies on variability of soil fertility, crop yields and nutrient balances on smallholder farms in Zimbabwe. Agriculture, Ecosystems and Environment. 2006; 119:112-126.

8. Bi L, Yao S, Zhang B. Impacts of long-term chemical and organic fertilization on soil puddlability in subtropical China. Soil Till Res. 2015; 152:94-103.

9. Fu W, Tunney $\mathrm{H}$, Zhang $\mathrm{C}$. Spatial variation of soil nutrients in a dairy farm and its implications for sitespecific fertilizer application. Soil Till Res. 2009; 106:185-193.

10. Garten CT, Kang S, Brice DJ, Schadt CW, Zhou J. Variability in soil properties at different spatial scales $(1 \mathrm{~m}-1 \mathrm{~km})$ in a deciduous forest ecosystem. Soil Biology and Biochemistry. 2007; 39:2621-2627.

11. Vasu D, Singh SK, Sahu N, Tiwary $P$, Chandran P, Duraisami VP, et al. Assessment of spatial variability of soil properties using geospatial techniques for farm level nutrient management. Soil Till Res. 2017; 169:25-34.

12. Rosemary F, Vitharana UWA, Indraratne SP, Weerasooriya R, Mishra U. Exploring the spatial variability of soil properties in an Alfisol soil catena. Catena. 2017; 150:53-61.

13. Paz A, Taboada MT, Gomez MJ. Spatial variability in topsoil micronutrient contents in a one-hectare cropland plot. Commun Soil Sci Plan. 1996; 27:479-503.

14. Yang Q, Yang J, Liu G. Scale-dependency of spatial variability of soil available nutrients. Chinese Journal of Applied Ecology 2011; 22:431-436. PMID: 21608258

15. Blanchet G, Libohova Z, Joost S, Rossier N, Schneider A, Jeangros B, et al. Spatial variability of potassium in agricultural soils of the canton of Fribourg, Switzerland. Geoderma. 2017; 290:107-121.

16. Bogunovic I, Mesic M, Zgorelec Z, Jurisic A, Bilandzija D. Spatial variation of soil nutrients on sandyloam soil. Soil Till Res. 2014; 144:174-183.

17. Shabani G, Ardakani MR, Chaichi MR, Friedel JK, Khavazi K. Effect of different fertilizing treatments on nutrient uptake in annual medic (Medicago scutellata cv. Robinson) under irrigated and dry farming systems. Journal of Agricultural Science \& Technology. 2015; 17:299-310.

18. Sanscartier D, Reimer K, Koch I, Laing T, Zeeb B. An Investigation of the Ability of a 14C-Labeled Hydrocarbon Mineralization Test to Predict Bioremediation of Soils Contaminated with Petroleum Hydrocarbons. Bioremediation Journal. 2009; 13:92-101.

19. Santillano-Cázares J, Redmon LA, Caddel JL, Goad CL, Rueda-Puente EO. Spatial and temporal variability of soil fertility in terraced pastures. J Plant Nutr. 2012; 35:2055-2066.

20. Qiao JF, Lai N, Geng QL, Li YL, Li Y, Yang H, et al. Spatial and temporal variation characteristics of soil nutrient accumulation in wheat of different drip irrigation years. Xinjiang Agricultural Sciences. 2017; 54:667-74.

21. Umali BP, Oliver DP, Forrester S, Chittleborough DJ, Hutson JL, Kookana RS, et al. The effect of terrain and management on the spatial variability of soil properties in an apple orchard. Catena. 2012; 93:3848.

22. Vaezi AR, Bahrami HA, Sadeghi SHR, Mahdian MH. Spatial variability of soil erodibility factor (K) of the USLE in north west of Iran. Journal of Agricultural Science \& Technology. 2010; 12:241-252.

23. Gallardo A, Paramá R. Spatial variability of soil elements in two plant communities of NW Spain. Geoderma. 2007; 139:199-208. 
24. Jackson-Blake L, Helliwell RC, Britton AJ, Gibbs S, Coull MC, Dawson L. Controls on soil solution nitrogen along an altitudinal gradient in the Scottish uplands. Sci Total Environ. 2012; 431:100-108. https:// doi.org/10.1016/j.scitotenv.2012.05.019 PMID: 22673176

25. Haileslassie A, Priess J, Veldkamp E, Teketay D, Lesschen JP. Assessment of soil nutrient depletion and its spatial variability on smallholders' mixed farming systems in Ethiopia using partial versus full nutrient balances. Agriculture, Ecosystems and Environment. 2004; 108:1-16.

26. Mohammadi J. Spatial Variability of Soil Fertility, Wheat Yield and Weed Density in a One-hectare Field in Shahre Kord. Journal of Agricultural Science \& Technology. 2002; 04:83-92.

27. Meynet P, Moliterni E, Davenport RJ, Sloan WT, Camacho JV, Werner D. Predicting the effects of biochar on volatile petroleum hydrocarbon biodegradation and emanation from soil: A bacterial community finger-print analysis inferred modelling approach. Soil Biology and Biochemistry. 2014; 68:20-30.

28. Cornfield AH. Ammonia released on Treating Soils with N Sodium Hydroxide as a Possible Means of predicting the Nitrogen-supplying Power of Soils. Nature. 1960; 187:260-261.

29. Olsen SR, Cole CV, Watanabe FS, Dean LA. Estimation of available phosphorus in soils by extraction with sodium bicarbonate. Circular of United States Department of Agriculture, pp. 939. 1954.

30. Schollenberger CJ, Simon RH. Determination of Exchange Capacity and Exchangeable Bases in SoilAmmonium Acetate Method. Soil Sci. 1945; 59:13-24.

31. Hu KL, Wang SY, Li H, Huang F, Li BG. Spatial scaling effects on variability of soil organic matter and total nitrogen in suburban Beijing. Geoderma. 2014; 226-227:54-63.

32. Zhang SW, Ge C, Chen XH, Li Z, Shen Q, Zhang LL, et al. Spatial distribution characteristics and scale effects of regional soil organic carbon. Transactions of the Chinese Society of Agricultural Engineering. 2018; 34:159-168.

33. Cambardella CA. Field-scale variability of soil properties in central lowa soils. Soilence Society of America Journal. 1994; 58:1501-1511.

34. Xu Y, Cai SH. The Spatio-temporal variability of soil available nutrients at field scale during the growing season of winter wheat. Journal of Shenyang Agricultural University. 2009; 40:296-300.

35. Wang CR, Lv JL, Hu ZY, Gao YM. Spatial varaibility and distribution pattern of soil nitrogen, phosphorus and potassium in vegetable field in typical area of taihu lake watershed. Chinese Agricultural Science Bulletin. 2005; 8:238-242.

36. Li YL, Zhao GX. Spatial prediction of cultivated land soil nutrients in typical region of yellow river delta. Journal of Natural Resources. 2018; 33:489-503.

37. He XB, Wang XQ, Ge SH, Li PY, Chang D. Study on spatial-temporal variability of available iron and manganese in soil during tobacco growth season. Shandong Agricultural Sciences. 2018; 50:101-106.

38. Miao YF, Li YJ, Zhang HM, Chen HJ, Chen MC. Influence of nitrogen and potassium fertilizers on nutrient assimilation of wheat and yield increase effect. Journal of Northwest A\&F University. 1999; 2:4650

39. $\mathrm{Xu} Y$, Kong $\mathrm{D}$. Relationships between yield and spatial variability of soil water and nutrient contents at different growing stages of winter wheat. Journal of Irrigation and Drainage. 2009; 28:27-30. 